\title{
AUnif: A COMMON SUPERCATEGORY OF PMET AND Unif
}

\author{
R. LOWEN
}

B. WINDELS

Department of Mathematics and Computer Science

University of Antwerp, RUCA

Groenenborgerlaan 171, 2020 Antwerp, BELGIUM

(Received July 17, 1996 and in revised form February 24, 1997)

\begin{abstract}
In order to have a topological category that contains both pMET and Unif in a nice way (and therefore combines quantitative and uniform concepts), approach uniformities are introduced. Approach uniformities uniformize the so-called approach spaces, introduced in Lowen [8]. Different characterizations of approach uniformities are formulated. Some natural examples are presented, such as function spaces, hyperspaces, spaces of measures, and an example concerning theoretical computer science.
\end{abstract}

KEY WORDS AND PHRASES: approach space, approach uniformity, uniform space. 1991 AMS SUBJECT CLASSIFICATION CODES: 54E15, 54B30

\section{INTRODUCTION}

Different results concerning completeness in the category AP (see Lowen [9]), imply that the theory is local. For instance, the space of continuous functions between metric spaces, equipped with the pointwise distance, turns out to be complete.

Also, AP seems not to be the right context for the quantification of uniform properties, such as completeness and total boundedness.

Therefore, it is natural to seek a new category that combines the quantitative aspects of AP (or pMET) and uniform concepts.

Recall that an approach space is a set $X$ equipped with a distance $\delta$, i.e. a map

$$
\delta: X \times 2^{X} \rightarrow[0, \infty]:(x, A) \mapsto \delta(x, A)
$$

satisfying the following conditions:

(D1) $\forall x \in X: \delta(x,\{x\})=0$

(D2) $\forall x \in X: \delta(x, \emptyset)=\infty$

(D3) $\forall x \in X, \forall A, B \subset X: \delta(x, A \cup B)=\min \{\delta(x, A), \delta(x, B)\}$

(D4) $\forall x \in X, \forall A \subset X, \forall \varepsilon \in[0, \infty]: \delta(x, A) \leq \delta\left(x, A^{(\varepsilon)}\right)+\varepsilon$ 
This description of approach spaces is intuitively the most appealing: the value $\delta(x, A)$ is interpreted as a distance from the point $x$ to the set $A$.

For the sake of uniformizing the concept of approach spaces, let us consider the following equivalent characterizations.

A collection of ideals $(\mathcal{A}(x))_{x \in X}$ in $[0, \infty]^{X}$ is called an approach system on $X$ iff for all $x \in X$ the following conditions are satisfied:

(A1) $\forall \varphi \in \mathcal{A}(x): \varphi(x)=0$

(A2) $\forall \varphi \in[0, \infty]^{X}:\left(\forall \varepsilon>0, \forall N<\infty: \exists \varphi_{\varepsilon}^{N} \in \mathcal{A}(x)\right.$ such that $\left.\varphi \wedge N \leq \varphi_{\varepsilon}^{N}+\varepsilon\right) \Rightarrow \varphi \in \mathcal{A}(x)$

(A3) $\forall \varphi \in \mathcal{A}(x), \forall \varepsilon>0, \forall N<\infty, \exists\left(\varphi_{z}\right)_{z \in X} \in \prod_{z \in X} \mathcal{A}(z)$ such that $\forall y, z \in X: \varphi(y) \wedge N \leq$ $\varphi_{x}(z)+\varphi_{z}(y)+\varepsilon$.

The value $\varphi(y)$ of a so-called local distance $\varphi \in \mathcal{A}(x)$ in a point $y \in X$, is interpreted as the distance from $x$ to $y$ according to $\varphi$.

A family of functions $\left(t_{\varepsilon}: 2^{X} \rightarrow 2^{X}\right)_{\varepsilon \in \mathbf{R}^{+}}$of pretopological closure operators is called a tower on $X$ iff the following conditions are satisfied:

(T1) $\forall A \in 2^{X}, \forall \varepsilon, \gamma \in \mathbf{R}^{+}: t_{\varepsilon}\left(t_{\gamma}(A)\right) \subset t_{\varepsilon+\gamma}(A)$

(T2) $\forall A \in 2^{X}, \forall \varepsilon \in \mathbf{R}^{+}: t_{\varepsilon}(A)=\bigcap_{\gamma>\varepsilon} t_{\gamma}(A)$.

An approach space $(X, \delta)$ if called a uniform approach space if there exists a collection of $\infty$-metrics $\mathcal{D}$ such that $\forall x \in X, \forall A \subset X: \delta(x, A)=\sup _{d \in D} \inf _{a \in A} d(x, a)$.

A function $f:(X, \delta) \rightarrow(Y, \eta)$ is called a contraction iff $\eta \circ(f \times f) \leq \delta$. The topological category of approach spaces together with contractions is denoted by AP. Its full subcategory of uniform approach spaces is denoted by UAP.

\section{APPROACH UNIFORMITIES}

\subsection{UNIFORM APPROACH SYSTEMS AND TOWERS}

In this section, we shall give two descriptions of what an approach uniformity might look like: the uniform approach system (a uniformization of approach systems) and the uniform tower (the uniform counterpart of approach towers). Then we shall prove that there is a one-one correspondence between both kind of structures. The reader may skip the proofs in this section.

If $\gamma \in[0, \infty]^{X \times X}$, then define for all $x, y \in X: \gamma^{s}(x, y):=\gamma(y, x)$.

DEFINITION 2.1. Let $X$ be a set. $A$ uniform approach system on $X$ is an ideal $\Gamma \subset$ $[0, \infty]^{X \times X}$ such that

(AU1) $\forall \gamma \in \Gamma, \forall x \in X: \gamma(x, x)=0$

(AU2) $\forall \xi \in[0, \infty]^{X \times X}:\left(\forall \varepsilon>0, \forall N<\infty: \exists \gamma_{\varepsilon}^{N} \in \Gamma\right.$ s.t. $\left.\xi \wedge N \leq \gamma_{\varepsilon}^{N}+\varepsilon\right) \Rightarrow \xi \in \Gamma$

(AU3) $\forall \gamma \in \Gamma, \forall N<\infty, \exists \gamma^{N} \in \Gamma$ s.t. $\forall x, y, z \in X: \gamma(x, z) \wedge N \leq \gamma^{N}(x, y)+\gamma^{N}(y, z)$

(AU4) $\forall \gamma \in \Gamma: \gamma^{s} \in \Gamma$. 
An ideal $\Gamma \subset[0, \infty]^{X \times X}$ satisfying (AU1), (AU2) and (AU3) shall be called an approach quasi-uniformity.

If $U \subset X \times X$ then $U^{-1}:=\{(y, x) \mid(x, y) \in U\}$ and $\Delta_{X}:=\{(x, x) \mid x \in X\}$.

DEFINITION 2.2. Let $X$ be a set. $A$ uniform tower on $X$ is a family of filters $\left(\mathcal{U}_{\varepsilon}\right)_{\varepsilon \in \mathbf{R}^{+}}$ on $X \times X$, such that

(UT1) $\forall \varepsilon \in \mathbf{R}^{+}, \forall U \in \mathcal{U}_{\varepsilon}: \Delta_{\boldsymbol{X}} \subset U$

(UT2) $\forall \varepsilon \in \mathbf{R}^{+}, \forall U \in \mathcal{U}_{\varepsilon}: U^{-1} \in \mathcal{U}_{\varepsilon}$

(UT3) $\forall \varepsilon, \varepsilon^{\prime} \in \mathbf{R}^{+}: \mathcal{U}_{\varepsilon} \circ \mathcal{U}_{\varepsilon^{\prime}} \supset \mathcal{U}_{\varepsilon+\varepsilon^{\prime}}$

(UT4) $\forall \varepsilon \in \mathbf{R}^{+}: \mathcal{U}_{\varepsilon}=\bigcup_{\alpha>\varepsilon} \mathcal{U}_{\alpha}$.

Thus, a uniform tower is a stack of semi-uniform spaces satisfying (UT3) and (UT4). Also notice that by (UT3), $U_{0}$ is a uniformity.

A family of filters $\left(\mathcal{U}_{\varepsilon}\right)_{\varepsilon} \in \mathrm{R}^{+}$on $X \times X$ satisfying (UT1), (UT3) and (UT4) shall be called a quasi-uniform tower.

In the sequel we shall show that there exists a one-one correspondence between uniform approach systems and uniform towers. A set $X$, equipped with one of these structures will be called an approach uniform space. Analogously, a set $X$ equipped with a quasi-uniform approach system or a quasi-uniform tower, will be called an approach quasi-uniform space.

But firstly we shall investigate what is the relationship between approach uniformities and ordinary approach spaces. Next proposition shows that an approach uniformity induces an approach space in a natural way.

PROPOSITION 2.3. Let $\Gamma$ be a quasi-uniform approach system on a set $X$. Then the family $(\mathcal{A}(x))_{x \in X}$, where

$$
\mathcal{A}(x)=\{\gamma(x, \cdot) \mid \gamma \in \Gamma\}
$$

is an approach system on $X$.

PROOF. We shall show (A2) and (A3).

(A2) Let $\varphi \in[0, \infty]^{X}$ be such that $\forall \varepsilon>0, \forall N<\infty, \exists \varphi_{\varepsilon}^{N} \in \mathcal{A}(x): \varphi \wedge N \leq \varphi_{\varepsilon}^{N}+\varepsilon$. Then for each $\varepsilon>0$ and $N<\infty$ there is some $\gamma_{\varepsilon}^{N} \in \Gamma$ such that $\varphi_{\varepsilon}^{N}=\gamma_{\varepsilon}^{N}(x, \cdot)$. Define

$$
\xi: X \times X \rightarrow[0, \infty]: \begin{cases}(z, y) \mapsto \varphi(y) & \text { if } z=x \\ (z, y) \mapsto 0 & \text { if } z \neq x\end{cases}
$$

For all $\varepsilon>0$ and $N<\infty$ we have $\forall y \in X$ :

$$
\begin{aligned}
\xi(x, y) \wedge N & =\varphi(y) \wedge N \\
& \leq \varphi_{\varepsilon}^{N}(y)+\varepsilon \\
& =\gamma_{\varepsilon}^{N}(x, y)+\varepsilon
\end{aligned}
$$

and for all $z \neq x$ and $y \in X$ by definition

$$
\xi(z, y) \wedge N \leq \gamma_{\varepsilon}^{N}(z, y)+\varepsilon .
$$

By (AU2), $\xi \in \Gamma$. Thus $\varphi=\xi(x, \cdot) \in \mathcal{A}(x)$. 
(A3) Let $\varphi \in \mathcal{A}(x)$, let $\varepsilon>0$ and $N<\infty$. Choose $\gamma \in \Gamma$ such that $\varphi=\gamma(x, \cdot)$ and choose $\gamma^{N} \in \Gamma$ such that

$$
\forall x, y, z \in X: \gamma(x, y) \wedge N \leq \gamma^{N}(x, z)+\gamma^{N}(z, y) .
$$

Define for each $z \in X$ the function $\varphi_{z}:=\gamma^{N}(z, \cdot)$. Then we have $\forall y, z \in X$ :

$$
\begin{aligned}
\varphi(y) \wedge N & =\gamma(x, y) \wedge N \\
& \leq \gamma^{N}(x, z)+\gamma^{N}(z, y) \\
& =\varphi_{x}(z)+\varphi_{z}(y) .
\end{aligned}
$$

If $\left(\mathcal{U}_{\varepsilon}\right)_{\varepsilon \in \mathbf{R}^{+}}$is a quasi-uniform tower, then each semi-quasi-uniformity $\mathcal{U}_{\varepsilon}$ induces a pretopological closure operator $t_{\varepsilon}$, yielding a tower $\left(t_{\varepsilon}\right)_{\varepsilon \in R^{+}}$. It is shown later in the text that, if $\left(\mathcal{U}_{\varepsilon}\right)_{\varepsilon \in \mathbf{R}^{+}}$and $\Gamma$ describe the same approach uniformity, then the tower $\left(t_{\varepsilon}\right)_{\varepsilon \in \mathbf{R}^{+}}$and the approach system in the previous proposition define the same approach structure, which will be denoted by $A(\Gamma)$.

Before we can move on to the proof of the equivalence of (quasi-) uniform towers and (quasi)uniform approach systems, we require some information about the basis of a (quasi-)uniform approach system. We start with the following proposition.

PROPOSITION 2.4. If $\Gamma \subset[0, \infty]^{X \times X}$ is an ideal, then the following are equivalent.

(AU2) $\forall \gamma \in \Gamma, \forall N<\infty, \exists \gamma^{N} \in \Gamma$ s.t. $\forall x, y, z \in X: \gamma(x, z) \wedge N \leq \gamma^{N}(x, y)+\gamma^{N}(y, z)$

(AU2') $\forall \gamma \in \Gamma, \forall N<\infty, \forall \varepsilon>0, \exists \gamma_{\varepsilon}^{N} \in \Gamma$ s.t. $\forall x, y, z \in X: \gamma(x, z) \wedge N \leq \gamma_{\varepsilon}^{N}(x, y)+\gamma_{\varepsilon}^{N}(y, z)+\varepsilon$.

PROOF. We only need to show that (AU2') $\Rightarrow$ (AU2). To that end, let $\gamma \in \Gamma$ and $N<\infty$. For each $n \in \mathbf{N}_{0}$, choose $\gamma_{n}^{N} \in \Gamma$ such that

$$
\forall x, y, z \in X: \gamma(x, z) \wedge N \leq \gamma_{n}^{N}(x, y)+\gamma_{n}^{N}(y, z)+\frac{1}{n} .
$$

Define $\gamma^{N}$ by

$$
\forall x, y \in X: \gamma^{N}(x, y)=\inf _{n \in N_{0}}\left(\sup _{k=1}^{n} \gamma_{k}^{N}(x, y)+\frac{1}{n}\right) .
$$

From (AU2) we deduce that $\gamma^{N} \in \Gamma$. Further, we have that

$$
\begin{aligned}
\gamma^{N}(x, y)+\gamma^{N}(y, z) & =\inf _{n \in \mathbb{N}_{0}} \inf _{m \in \mathbb{N}_{0}}\left(\sup _{k=1}^{n} \gamma_{k}^{N}(x, y)+\frac{1}{n}+\sup _{j=1}^{m} \gamma_{j}^{N}(y, z)+\frac{1}{m}\right) \\
& \geq \inf _{n \in N_{0}}\left(\sup _{k=1}^{n} \gamma_{k}^{N}(x, y)+\sup _{j=1}^{n} \gamma_{j}^{N}(y, z)+\frac{1}{n}\right) \\
& \geq \inf _{n \in \mathbb{N}_{0}}\left(\sup _{k=1}^{n}\left(\gamma_{k}^{N}(x, y)+\gamma_{k}^{N}(y, z)\right)+\frac{1}{n}\right) \\
& \geq \inf _{n \in \mathbb{N}_{0}}\left(\sup _{k=1}^{n}\left(\gamma(x, z) \wedge N-\frac{1}{k}\right)+\frac{1}{n}\right) \\
& =\gamma(x, z) \wedge N .
\end{aligned}
$$

DEFINITION 2.5. Let $X$ be a set and let $\Gamma \subset[0, \infty]^{X \times X}$. Then $\Gamma$ is called a uniform approach basis on $X$ iff $\Gamma$ is an ideal basis (i.e.: $\forall \gamma_{1}, \gamma_{2} \in \Gamma: \exists \gamma_{3} \in \Gamma$ s.t. $\gamma_{1} \vee \gamma_{2} \leq \gamma_{3}$ ) satisfying (AU1), (AU3) and (AU4).

If $\Gamma$ is a uniform approach basis, then

$$
\langle\Gamma\rangle:=\left\{\gamma \in[0, \infty]^{X \times X} \mid \forall \varepsilon>0, \forall N<\infty, \exists \gamma_{\varepsilon}^{N} \in \Gamma \text { s.t. } \gamma \wedge N \leq \gamma_{\varepsilon}^{N}+\varepsilon\right\}
$$


will be called the saturation of $\Gamma$.

If $\Psi$ is a uniform approach system and $\Gamma$ is a uniform approach basis such that $\Psi=\langle\Gamma\rangle$, then $\Gamma$ is called a basis for $\Psi$.

PROPOSITION 2.6. If $\Gamma$ is a uniform approach basis, then $\langle\Gamma\rangle$ is a uniform approach system with $\Gamma$ as a basis.

PROOF. By saturation it is immediately clear that $\langle\Gamma\rangle$ is an ideal. We only have to show that $\langle\Gamma\rangle$ satisfies (AU1) - (AU4).

(AU1) If $\gamma \in\langle\Gamma\rangle$, then $\forall \varepsilon>0, \exists \gamma_{\varepsilon}^{1} \in \Gamma$ such that $\gamma \wedge 1 \leq \gamma_{\varepsilon}^{1}+\varepsilon$. Thus $\forall x \in X, \forall \varepsilon>0$ : $\gamma(x, x) \wedge 1 \leq \gamma_{\varepsilon}^{1}(x, x)+\varepsilon=\varepsilon$. Therefore $\forall x \in X: \gamma(x, x)=0$.

(AU2) Let $\xi \in[0, \infty]^{X \times X}$ be such that

$$
\forall \varepsilon>0, \forall N<\infty, \exists \gamma_{\varepsilon}^{N} \in\langle\Gamma\rangle: \xi \wedge N \leq \gamma_{\varepsilon}^{N}+\frac{\varepsilon}{2} .
$$

Fix $\varepsilon>0$ and $N<\infty$. Then there is some $\psi \in \Gamma$ such that $\gamma_{\varepsilon}^{N} \wedge N \leq \psi+\frac{\varepsilon}{2}$ and consequently

$$
\begin{aligned}
\xi \wedge N & \leq \gamma_{\varepsilon}^{N} \wedge N+\frac{\varepsilon}{2} \\
& \leq \psi+\varepsilon .
\end{aligned}
$$

(AU3) Let $\gamma \in\langle\Gamma\rangle, \varepsilon>0$ and $N<\infty$. Then there is some $\gamma_{\varepsilon}^{N} \in \Gamma$ such that $\gamma \wedge N \leq \gamma_{\varepsilon}^{N}+\frac{\varepsilon}{2}$, and there is some $\psi_{\varepsilon}^{N} \in \Gamma$ such that

$$
\forall x, y, z \in X: \gamma_{\varepsilon}^{N}(x, z) \wedge N \leq \psi_{\varepsilon}^{N}(x, y)+\psi_{\varepsilon}^{N}(y, z)+\frac{\varepsilon}{2} .
$$

Consequently

$$
\begin{aligned}
\gamma(x, z) \wedge N & \leq \gamma_{\varepsilon}^{N}(x, z) \wedge N+\frac{\varepsilon}{2} \\
& \leq \psi_{\varepsilon}^{N}(x, y)+\psi_{\varepsilon}^{N}(y, z)+\varepsilon
\end{aligned}
$$

(AU4) If $\gamma \in \Gamma$ then obviously

$$
\forall \varepsilon>0, \forall N<\infty, \exists \gamma_{\varepsilon}^{N} \in \Gamma: \gamma^{s} \wedge N \leq\left(\gamma_{\varepsilon}^{N}\right)^{s}+\varepsilon
$$

whence $\gamma^{s} \in\langle\Gamma\rangle$.

We now turn to a lemma that will be of paramount importance in proving the one-one relationship between systems and towers.

For any $N<\infty$, we define a ( $\delta$-) net on $[0, N]$ to be a finite collection $\left\{\alpha_{0}, \ldots, \alpha_{n}\right\}$ such that $\alpha_{0}=0, \alpha_{n}=N$ and $\forall i \in\{1, \ldots, n\}: \alpha_{i}-\alpha_{i-1}=\delta=N / n$.

If $U \subset X \times X$, then we define

$$
\theta_{U}: X \times X \rightarrow\{0, \infty\}: \begin{cases}(x, y) \mapsto 0 & \text { if }(x, y) \in U \\ (x, y) \mapsto \infty & \text { otherwise. }\end{cases}
$$

If $\gamma \in[0, \infty]^{X \times X}$ and $\alpha>0$ then we write $\{\gamma<\alpha\}:=\{(x, y) \in X \times X \mid \gamma(x, y)<\alpha\}$ for short.

LEMMA 2.7. Let $\left(\mathcal{U}_{\varepsilon}\right)_{\varepsilon}$ be a quasi-uniform tower. If

$$
\Gamma:=\left\{\gamma \in[0, \infty]^{X \times X} \mid \forall \varepsilon \in \mathbf{R}^{+}, \forall \alpha>\varepsilon:\{\gamma<\alpha\} \in \mathcal{U}_{\varepsilon}\right\}
$$


and

$$
\Psi:=\left\{\begin{array}{ll}
\inf _{i=1}^{n}\left(\alpha_{i-1}+\theta_{U_{1}}\right) \wedge N \mid \begin{array}{l}
N<\infty, \delta>0,\left\{\alpha_{0}, \ldots, \alpha_{n}\right\} \text { a } \delta \text {-net on }[0, N], \\
\forall i \in\{1, \ldots, n\}: U_{i} \in \mathcal{U}_{\alpha_{1}} \text { and } U_{i-1} \subset U_{i}
\end{array}
\end{array}\right\}
$$

then $\Gamma=\langle\Psi\rangle$.

PROOF. Firstly we show that $\Psi \subset \Gamma$, and therefore $\langle\Psi\rangle \subset\langle\Gamma\rangle$. Suppose $\gamma=\inf _{i=1}^{n}\left(\alpha_{s-1}+\right.$ $\left.\theta_{U_{\imath}}\right) \wedge N \in \Psi$ and let $\varepsilon \in \mathbf{R}^{+}$and $\alpha>\varepsilon$. If $\varepsilon \geq N$, then $\{\gamma<\alpha\}=X \times X \in \mathcal{U}_{\varepsilon}$. If $\varepsilon \in\left[\alpha_{1-1}, \alpha_{2}[\right.$ for some $i \in\{1, \ldots, n\}$, then $\{\gamma<\alpha\} \supset U_{i} \in \mathcal{U}_{\alpha} \subset \mathcal{U}_{\varepsilon}$, whence $\{\gamma<\alpha\} \in \mathcal{U}_{\varepsilon}$.

Secondly, we show that $\Gamma$ is saturated, and therefore $\langle\Psi\rangle \subset \Gamma$. Let $\xi \in\langle\Gamma\rangle$. Fix $\varepsilon \in \mathbf{R}^{+}$and $\alpha>\varepsilon$. Then there exists some $\gamma \in \Gamma$ such that

$$
\xi \wedge \alpha \leq \gamma+\frac{\alpha-\varepsilon}{2}
$$

Then we have $\forall x, y \in X$ :

$$
\begin{aligned}
\gamma(x, y)<\frac{\alpha+\varepsilon}{2} & \Rightarrow \gamma(x, y)+\frac{\alpha-\varepsilon}{2}<\alpha \\
& \Rightarrow \xi(x, y) \wedge \alpha<\alpha \\
& \Rightarrow \xi(x, y)<\alpha .
\end{aligned}
$$

Therefore $\{\xi<\alpha\} \supset\left\{\gamma<\frac{\varepsilon+\alpha}{2}\right\} \in \mathcal{U}_{\varepsilon}$, whence $\{\xi<\alpha\} \in \mathcal{U}_{\varepsilon}$. Hence, $\xi \in \Gamma$.

Finally, we shall show that $\Gamma \subset\langle\Psi\rangle$. To this end, we shall prove that for any $\xi \in \Gamma$, and for any $\varepsilon>0$ and $N<\infty$, there is some $\gamma_{\varepsilon}^{N} \in \Psi$ such that $\xi \wedge N \leq \gamma_{\varepsilon}^{N}+\varepsilon$. Fix $\xi \in \Gamma, \varepsilon>0$ and $N<\infty$, and some $\delta$-net $\left\{\alpha_{0}, \ldots, \alpha_{n}\right\}$ on $[0, N]$ such that $\delta<\varepsilon / 2$. Put $\forall i \in\{1, \ldots, n\}: U_{i}:=$ $\left\{\xi<\alpha_{i}+\delta / 2\right\} \in \mathcal{U}_{\alpha_{i}}$ and

$$
\gamma_{\varepsilon}^{N}:=\inf _{i=1}^{n}\left(\alpha_{i-1}+\theta_{U_{i}}\right) \wedge N .
$$

Let $x, y \in X$. If $\gamma_{\varepsilon}^{N}(x, y)+\varepsilon \geq N$, then there is nothing to prove. Suppose $\gamma_{\varepsilon}^{N}(x, y)+\varepsilon \leq A \in$ $\left[\alpha_{i}, \alpha_{i+1}[\right.$ for some $i \in\{0, \ldots, n-1\}$. Then

$$
\begin{aligned}
& \gamma_{\varepsilon}^{N}(x, y)+2 \delta \leq A \in\left[\alpha_{i}, \alpha_{i+1}\right] \\
\Rightarrow & \gamma_{\varepsilon}^{N}(x, y) \leq A-2 \delta \in\left[\alpha_{i-2}, \alpha_{i-1}\right] \\
\Rightarrow & (x, y) \in U_{i-1} \\
\Rightarrow & \xi(x, y)<\alpha_{i-1}+\delta / 2 \leq \alpha_{i} \leq A .
\end{aligned}
$$

Thus $\xi \wedge N \leq \gamma_{\varepsilon}^{N}+\varepsilon$.

PROPOSITION 2.8. If $\left(U_{\varepsilon}\right)_{\varepsilon \in R^{+}}$is a (quasi-)uniform tower, then

$$
\Gamma:=\left\{\gamma \in[0, \infty]^{X \times X} \mid \forall \varepsilon \in \mathbf{R}^{+}, \forall \alpha>\varepsilon:\{\gamma<\alpha\} \in \mathcal{U}_{\varepsilon}\right\}
$$

is a (quasi-)uniform approach system.

PROOF. By proposition 2.6 it suffices to show that $\Psi$ (defined as in lemma 2.7 ) satisfies (AU2'). Let

$$
\gamma:=\inf _{j=1}^{k}\left(\alpha_{j-1}+\theta_{U}\right) \wedge N \in \Psi .
$$

Since $\gamma$ is bounded, it suffices to prove that

$$
\forall \varepsilon>0: \exists \gamma_{\varepsilon} \in \Psi: \forall x, y, z \in X: \gamma(x, z) \leq \gamma_{\varepsilon}(x, y)+\gamma_{\varepsilon}(y, z)+\varepsilon .
$$


Fix $\varepsilon>0$ and $x, y, z \in X$. Refine the net $\left\{\alpha_{0}, \ldots, \alpha_{k}\right\}$ into a $\delta$-net $\left\{\beta_{0}, \ldots, \beta_{k n}\right\}$ on $[0, N]$ such that

$$
\forall j \in\{0, \ldots, k\}: \beta_{\jmath^{n}}=\alpha_{j} \text { and } \delta<\varepsilon / 2 .
$$

If $j \in\{1, \ldots, k\}$, then $\forall \beta_{p}, \beta_{q}$ such that $\beta_{p}+\beta_{q}=\alpha_{\jmath}$ and $\beta_{p} \in\left[0, \alpha_{\jmath} / 2\right]$, there are $V_{p}^{\jmath} \in \mathcal{U}_{\beta_{p}}$ and $V_{q}^{j} \in \mathcal{U}_{\beta_{q}}$ such that $V_{p}^{j} \circ V_{q}^{j} \subset U_{j}$. Define $\forall p \in\{1, \ldots, k n\}$ :

$$
V_{p}:=\bigcap\left\{V_{p}^{j} \mid j \in\{1, \ldots, k\}, \beta_{p} \leq \alpha_{j}\right\}
$$

and

Notice that $W_{p} \in \mathcal{U}_{\beta_{p}}$. Define

$$
W_{p}:=\bigcap_{q \geq p} V_{q}
$$

$$
\gamma_{\varepsilon}:=\inf _{i=1}^{k n}\left(\beta_{i-1}+\theta_{W_{2}}\right) \wedge N \in \Psi .
$$

Now suppose that $(x, z) \notin U_{1}$, otherwise there is nothing to prove. Then $\gamma(x, z)=\alpha_{j}$ for some $j \in\{1, \ldots, k\}$. If $\gamma_{\varepsilon}(x, y) \geq \alpha_{j}$ or $\gamma_{\varepsilon}(y, z) \geq \alpha_{j}$, then again there is nothing to prove. Therefore suppose that $\gamma_{\varepsilon}(x, y)=\beta_{p-1}(p \leq j n)$ and $\gamma_{\varepsilon}(y, z)=\beta_{q-1}(q \leq j n)$. Now suppose that $\beta_{p-1}+\beta_{q-1}+\varepsilon<\alpha_{j}$. Then

$$
\begin{aligned}
\beta_{p}+\beta_{q} & =\beta_{p-1}+\beta_{q-1}+2 \delta \\
& <\beta_{p-1}+\beta_{q-1}+\varepsilon \\
& <\alpha_{g} .
\end{aligned}
$$

Then there would exist some $r>p$ such that $\beta_{r}+\beta_{q}=\alpha_{j}$. Since $\beta_{r} \leq \alpha$, and $\beta_{q} \leq \alpha_{j}$, we have

$$
(x, y) \in W_{p} \subset W_{r} \subset V_{r} \subset V_{r}^{j}
$$

and

$$
(y, z) \in W_{q} \subset V_{q} \subset V_{q}^{3}
$$

and thus $(x, z) \in U_{j}$. Hence, $\gamma(x, z) \leq \alpha_{j-1}$, which is a contradiction. Therefore $\beta_{p-1}+\beta_{q-1}+\varepsilon \geq$ $\alpha_{j}$, which is exactly what we had to prove.

If $\Gamma$ is the (quasi-) uniform approach system induced by a (quasi-) uniform tower $\left(\mathcal{U}_{\varepsilon}\right)_{\varepsilon}$, then we denote this by $\Gamma=T_{t a}\left(\left(\mathcal{U}_{\varepsilon}\right)_{\varepsilon}\right)$.

If $\mathcal{F}$ is a filter basis then we denote $\langle\mathcal{F}\rangle:=$ stack $\mathcal{F}$.

PROPOSITION 2.9. If $\Gamma$ is a (quasi-) uniform approach system, then the family $\left(\mathcal{U}_{\varepsilon}\right)_{\varepsilon \in \mathbb{R}^{+}}$, defined by $\forall \varepsilon \in \mathbf{R}^{+}$:

$$
\mathcal{U}_{\varepsilon}:=\langle\{\{\gamma<\alpha\} \mid \gamma \in \Gamma, \alpha>\varepsilon\}\rangle
$$

is a (quasi-) uniform tower.

PROOF. We shall only prove (UT3) and (UT4).

In order to prove (UT3), fix $\varepsilon \in \mathbf{R}^{+}, \alpha>\varepsilon, \gamma \in \Gamma$, and $\varepsilon_{1}, \varepsilon_{2} \in \mathbf{R}^{+}$such that $\varepsilon_{1}+\varepsilon_{2}=\varepsilon$. We have to show that there exist $\gamma^{\prime} \in \Gamma, \alpha_{1}>\varepsilon_{1}, \alpha_{2}>\varepsilon_{2}$ such that

$$
\left\{\gamma^{\prime}<\alpha_{1}\right\} \circ\left\{\gamma^{\prime}<\alpha_{2}\right\} \subset\{\gamma<\alpha\}
$$

To this end, pick $\alpha_{1}>\varepsilon_{1}$ and $\alpha_{2}>\varepsilon_{2}$ such that $\alpha_{1}+\alpha_{2}=\alpha$, and take $\gamma^{\prime} \in \Gamma$ such that $\forall x, y, z \in X: \gamma(x, z) \wedge \alpha \leq \gamma^{\prime}(x, y)+\gamma^{\prime}(y, z)$. 
To prove (UT4), notice that if $\{\gamma<\alpha\} \in \mathcal{U}_{\varepsilon}$, then $\{\gamma<\alpha\} \in \mathcal{U}_{\mu}$ for all $\left.\mu \in\right] \varepsilon, \alpha$. Hence $\mathcal{U}_{\varepsilon} \subset U_{\mu>\varepsilon} \mathcal{U}_{\mu}$.

Conversely, if $\{\gamma<\alpha\} \in \mathcal{U}_{\mu}$ for some $\mu>\varepsilon$, then clearly $\{\gamma<\alpha\} \in \mathcal{U}_{\varepsilon}$, and therefore $\bigcup_{\mu>\varepsilon} \mathcal{U}_{\mu} \subset$ $\mathcal{U}_{\varepsilon}$.

If $\left(\mathcal{U}_{\varepsilon}\right)_{\varepsilon}$ is the (quasi-)uniform tower induced by a (quasi-)uniform system $\Gamma$, then we denote this by $\left(\mathcal{U}_{\varepsilon}\right)_{\varepsilon}=T_{\text {at }}(\Gamma)$.

The following propositions show that $T_{t a}$ and $T_{a t}$ define a one-one correspondence between uniform towers and uniform approach systems.

PROPOSITION 2.10. Let $\left(\mathcal{U}_{\varepsilon}\right)_{\varepsilon}$ be a quasi-uniform tower, and let $\left(\mathcal{V}_{\varepsilon}\right)_{\varepsilon}:=T_{t a} \circ T_{a t}\left(\left(\mathcal{U}_{\varepsilon}\right)_{\varepsilon}\right)$, then $\forall \varepsilon \in \mathbf{R}^{+}: \mathcal{U}_{\varepsilon}=\mathcal{V}_{\varepsilon}$.

PROOF. By definition we have $\forall \varepsilon \in \mathbf{R}^{+}$:

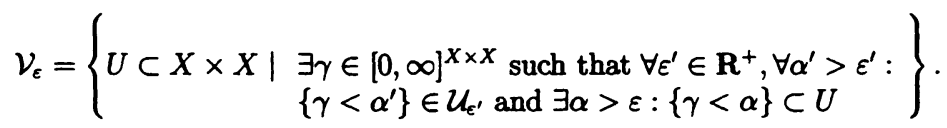

To prove that $\mathcal{V}_{\varepsilon} \subset \mathcal{U}_{\varepsilon}$, let $U \in \mathcal{V}_{\varepsilon}$. Then

(1) $\exists \gamma \in[0, \infty]^{X \times X}$ such that $\forall \varepsilon^{\prime} \in \mathbf{R}^{+}, \forall \alpha^{\prime}>\varepsilon^{\prime}:\left\{\gamma<\alpha^{\prime}\right\} \in \mathcal{U}_{\varepsilon^{\prime}}$

(2) $\exists \alpha>\varepsilon:\{\gamma<\alpha\} \subset U$

Let $\alpha$ be as in (2), then applying (1) for $\varepsilon^{\prime}:=\varepsilon$ and $\alpha^{\prime}:=\alpha$, yields $\{\gamma<\alpha\} \in \mathcal{U}_{\varepsilon}$, and thus, by (2), $U \in \mathcal{U}_{\varepsilon}$.

To prove that $\mathcal{U}_{\varepsilon} \subset \mathcal{V}_{\varepsilon}$, let $U \in \mathcal{U}_{\varepsilon}$. By (UT4), there is some $\delta>\varepsilon$ such that $U \in \mathcal{U}_{\delta}$. Define $\gamma:=\theta_{U} \wedge \delta$. Let $\varepsilon^{\prime}>0$, then if $\varepsilon^{\prime}>\delta$, we have $\forall \alpha^{\prime}>\varepsilon^{\prime}:\left\{\gamma<\alpha^{\prime}\right\}=X \times X \in \mathcal{U}_{\varepsilon^{\prime}}$, and if $\varepsilon^{\prime} \leq \delta$, then we have $\forall \alpha^{\prime}>\varepsilon^{\prime}:\left\{\gamma<\alpha^{\prime}\right\} \supset U \in \mathcal{U}_{\delta} \subset \mathcal{U}_{\varepsilon^{\prime}}$. Let $\alpha:=\frac{\varepsilon+\delta}{2}>\varepsilon$. Clearly we have that $\{\gamma<\alpha\}=U$.

PROPOSITION 2.11. Let $\Gamma$ be a quasi-uniform approach system, and let $\Psi:=T_{\text {at }} \circ T_{\mathrm{ta}}(\Gamma)$. Then $\Gamma=\Psi$.

PROOF. By definition, we have that

$$
\Psi=\left\{\psi \in[0, \infty]^{X \times X} \mid \forall \varepsilon \in \mathbf{R}^{+}, \forall \alpha^{\prime}>\varepsilon, \exists \gamma \in \Gamma, \exists \alpha>\varepsilon:\{\gamma<\alpha\} \subset\left\{\psi<\alpha^{\prime}\right\}\right\} .
$$

In order to prove that $\Gamma \subset \Psi$, let $\gamma^{\prime} \in \Gamma, \varepsilon^{\prime} \in \mathbf{R}^{+}$and $\alpha^{\prime}>\varepsilon$. If $\gamma:=\gamma^{\prime}$ and $\alpha:=\alpha^{\prime}$, we clearly have $\{\gamma<\alpha\} \subset\left\{\gamma^{\prime}<\alpha^{\prime}\right\}$. Therefore $\gamma^{\prime} \in \Psi$.

To prove that $\Psi \subset \Gamma$, suppose that $\left(\mathcal{U}_{\varepsilon}\right)_{\varepsilon}=T_{a t}(\Gamma)$. Then $\Psi$ has a basis consisting of functions of the form $\psi:=\inf _{i=1}^{n}\left(\alpha_{i-1}+\theta_{U_{i}}\right) \wedge N$ satisfying the conditions of lemma 2.7. If $i \in\{1, \ldots, n\}$ and $U_{i} \in \mathcal{U}_{\alpha_{i}}$, then there are $\gamma_{i} \in \Gamma$ and $\alpha_{i}^{+}>\alpha_{i}$ such that $\left\{\gamma_{i}<\alpha_{i}^{+}\right\} \subset U_{i}$. We shall show that $\psi \leq \bigvee_{i=1}^{n} \gamma_{i} \in \Gamma$, and therefore $\psi \in \Gamma$. Suppose $\psi(x, y)=\alpha_{i}$. Then $(x, y) \notin U_{i} \supset\left\{\gamma_{i}<\alpha_{i}^{+}\right\}$, thus $(x, y) \notin\left\{\gamma_{i}<\alpha_{i}^{+}\right\}$. Then $\gamma_{i}(x, y) \geq \alpha_{i}^{+}>\alpha_{i}$ and $\psi(x, y)<\gamma_{i}(x, y)$.

We already mentioned that every uniform approach system induces an ordinary approach system. On the other hand, every uniform tower has an underlying tower. These two underlying structures define the same approach space.

PROPOSITION 2.12. Let $\Gamma$ be a uniform approach system, and let $\left(\mathcal{U}_{\varepsilon}\right)_{\varepsilon}$ be the induced uniform tower. If $\left(t_{\varepsilon}\right)_{\varepsilon}$ is the tower defining $A(\Gamma)$, then $\forall \varepsilon \in \mathbf{R}^{+}, t_{\varepsilon}$ is the pre-closure operator induced by the semi-uniformity $\mathcal{U}_{\varepsilon}$, i.e. $\forall \varepsilon \in \mathbf{R}^{+}, \forall A \subset X: t_{\varepsilon}(A)=\bigcap_{U \in \mathcal{U}_{\varepsilon}} U(A)$. 
PROOF. Let $\varepsilon \in \mathbf{R}^{+}$and $A \subset X$. Then

$$
\begin{aligned}
t_{\varepsilon}(A) & =\left\{x \in X \mid \forall \varphi \in \mathcal{A}(x): \inf _{y \in A} \varphi(y) \leq \varepsilon\right\} \\
& =\left\{x \in X \mid \sup _{\gamma \in \Gamma} \inf _{y \in A} \gamma(x, y) \leq \varepsilon\right\} \\
& =\bigcap_{\gamma \in \Gamma}\{y \in X \mid \forall \alpha>\varepsilon, \exists x \in A: \gamma(x, y)<\alpha\} \\
& =\bigcap_{U \in \mathcal{U}_{e}}\{y \in X \mid \exists x \in A:(x, y) \in U\} \\
& =\bigcap_{U \in \mathcal{U}_{e}} U(A) .
\end{aligned}
$$

In the non-symmetric case, we have an analogous result. One easily verifies that $\forall \varepsilon \in$ $\mathbf{R}^{+}, \forall A \subset X: t_{\varepsilon}(A)=\bigcap_{U \in U_{\varepsilon}} U^{-1}(A)$.

\subsection{UNIFORM CONTRACTIONS}

DEFINITION 2.13. Let $(X, \Gamma)$ and $(Y, \Psi)$ be approach (quasi-)uniformities, and let $f: X \rightarrow Y$ be a function. Then $f:(X, \gamma) \rightarrow(Y, \Psi)$ is a uniform contraction iff $\forall \psi \in \Psi: \psi \circ(f \times f) \in \Gamma$.

PROPOSITION 2.14. Let $(X, \Gamma)$ and $(Y, \Psi)$ be approach (quasi-)uniformities, and let $\left(\mathcal{U}_{\varepsilon}\right)_{\varepsilon}$ and $\left(\mathcal{V}_{\varepsilon}\right)_{\varepsilon}$ be their (quasi-) uniform towers. Then the following are equivalent.

(1) $f$ is a uniform contraction

(2) $\forall \varepsilon \in \mathbf{R}^{+}: f:\left(X, \mathcal{U}_{\varepsilon}\right) \rightarrow\left(Y, \mathcal{V}_{\varepsilon}\right)$ is uniformly continuous.

PROOF. In order to prove that (1) $\Rightarrow(2)$, let $\varepsilon \in \mathbf{R}^{+}$and $V \in \mathcal{V}_{\varepsilon}$. Then there are $\psi \in \Psi$ and $\alpha>\varepsilon$ such that $\{\gamma<\alpha\} \subset V$. Since $\psi \circ(f \times f) \in \Gamma$, we have that $(f \times f)^{-1}(\{\gamma<\alpha\})=$ $\{\psi \circ(f \times f)<\alpha\} \in \mathcal{U}_{\varepsilon}$, and thus $(f \times f)^{-1}(V) \in \mathcal{U}_{\varepsilon}$.

To prove that (2) $\Rightarrow(1)$, let $\psi \in \Psi$. Then $\forall \varepsilon \in \mathbf{R}^{+}, \forall \alpha>\varepsilon:\{\psi<\alpha\} \in \mathcal{V}_{\varepsilon}$, and therefore $\{\psi \circ(f \times f)<\alpha\}=(f \times f)^{-1}(\{\psi<\alpha\}) \in \mathcal{U}_{\varepsilon}$, and thus $\psi \circ(f \times f) \in \Gamma$.

\subsection{THE TOPOLOGICAL CATEGORY AUnif}

One easily verifies that approach uniformities, together with uniform contractions form a category, which we shall denote as AUnif. The category of approach quasi-uniformities shall be denoted by AqUnif.

PROPOSITION 2.15. AUnif is a topological category.

PROOF. Let $\left(f_{j}: X \rightarrow\left(X_{j}, \Gamma_{j}\right)\right)_{j \in J}$ be a AUnif-source. Then the initial structure is

$$
\Gamma:=\left\{\sup _{j \in J_{0}} \gamma_{j} \circ\left(f_{j} \times f_{j}\right) \mid J_{0} \in 2^{(J)}, \forall j \in J_{0}: \gamma_{j} \in \Gamma_{j}\right\} .
$$

Initial structures can be described with uniform towers too. In fact, a AUnif-source is initial iff for any level $\varepsilon \in \mathbf{R}^{+}$the induced sUnif-source is initial.

PROPOSITION 2.16. Let $J$ be a set, let $\left(X,\left(\mathcal{U}_{\varepsilon}\right)_{\varepsilon}\right)$ and for all $j \in J,\left(Y_{j},\left(\mathcal{V}_{\varepsilon}^{j}\right)_{\varepsilon}\right)$ be approach uniformities, and let for all $j \in J, f_{j}: X \rightarrow Y_{j}$ be a function. Then the following are equivalent.

(1) $\left(f_{\jmath}:\left(X,\left(\mathcal{U}_{\varepsilon}\right)_{\varepsilon}\right) \rightarrow\left(Y_{\jmath},\left(\mathcal{V}_{\varepsilon}^{\mathcal{J}}\right)_{\varepsilon}\right)\right)_{j \in J}$ is initial.

(2) $\forall \varepsilon \in \mathbf{R}^{+}:\left(f_{j}:\left(X, \mathcal{U}_{\varepsilon}\right) \rightarrow\left(Y_{j}, \mathcal{V}_{\varepsilon}^{\jmath}\right)\right)_{j \in J}$ is initial. 
PROOF. Let $\mathcal{W}_{\varepsilon}$ denote the initial semi-uniformity for the source $\left(f_{j}: X \rightarrow\left(Y_{j}, \mathcal{V}_{\varepsilon}^{j}\right)\right)_{j \in J}$. Let $\Gamma=T_{t a}\left(\left(\mathcal{U}_{\varepsilon}\right)_{\varepsilon}\right)$ and $\Psi_{j}=T_{t a}\left(\left(\mathcal{V}_{\varepsilon}^{j}\right)_{\varepsilon}\right)$. We have $\mathcal{V}_{\varepsilon}^{j}=\left\langle\left\{\left\{\gamma_{j}<\alpha\right\} \mid \gamma_{j} \in \Psi_{j}, \alpha>\varepsilon\right\}\right)$, and thus

$$
\mathcal{W}_{\varepsilon}=\left\langle\left\{\bigcap_{\jmath \in J_{0}}\left(f_{\jmath} \times f_{j}\right)^{-1}\left(\left\{\gamma_{j}<\alpha_{\jmath}\right\}\right) \mid J_{0} \in 2^{(J)}, \gamma_{j} \in \Psi_{\jmath}, \alpha_{\jmath}>\varepsilon\right\}\right\rangle .
$$

We also have

$$
\Gamma:=\left\{\sup _{j \in J_{0}} \gamma_{j} \circ\left(f_{j} \times f_{j}\right) \mid J_{0} \in 2^{(J)}, \forall j \in J_{0}: \gamma_{j} \in \Gamma,\right\}
$$

and therefore

$$
\begin{aligned}
\mathcal{U}_{\varepsilon} & =\left\langle\left\{\left\{\sup _{j \in J_{0}} \gamma_{j} \circ\left(f_{j} \times f_{j}\right)<\alpha\right\} \mid J_{0} \in 2^{(J)}, \forall j \in J_{0}: \gamma_{j} \in \Gamma_{j}, \alpha>\varepsilon\right\}\right\rangle \\
& =\left\langle\left\{\bigcap_{j \in J_{0}}\left(f_{j} \times f_{j}\right)^{-1}\left(\left\{\gamma_{j}<\alpha\right\}\right) \mid J_{0} \in 2^{(J)}, \gamma_{j} \in \Psi_{j}, \alpha>\varepsilon\right\}\right\rangle .
\end{aligned}
$$

Clearly $\mathcal{W}_{\varepsilon}=\mathcal{U}_{\varepsilon}$.

We already mentioned that every approach uniformity has an underlying ordinary approach space. This operation in fact defines a forgetful functor. We need to show the following proposition.

PROPOSITION 2.17. If $f:(X, \Gamma) \rightarrow(Y, \Psi)$ is a uniform contraction, then $f:(X, A(\Gamma)) \rightarrow$ $(Y, A(\Psi))$ is a contraction.

PROOF. Let $(\mathcal{A}(x))_{x \in X}$ and $(\mathcal{B}(y))_{y \in Y}$ denote the approach systems of $A(\Gamma)$ and $A(\Psi)$ respectively. Let $x \in X$ and $\varphi \in \mathcal{B}(f(x))$. Then there is some $\psi \in \Psi$ such that $\varphi=\psi(f(x), \cdot)$. Then $\psi \circ(f \times f) \in \Gamma$ and therefore $\varphi \circ f=\psi(f(x), f(\cdot))=\psi \circ(f \times f)(x, \cdot) \in \mathcal{A}(x)$.

COROLLARY 2.18. $A:$ AqUnif $\rightarrow$ AP is a forgetful functor.

$$
\begin{array}{cccc}
(X, \Gamma) & \mapsto & (X, A(\Gamma)) \\
f & \mapsto & f
\end{array}
$$

\section{METRIC AND UNIFORM APPROACH UNIFORMITIES}

\subsection{METRIC APPROACH UNIFORMITIES}

Metric spaces can be interpreted as approach uniformities in the following manner.

PROPOSITION 3.1. Let $(X, d)$ be $a \infty p$-metric space. Then $\Gamma(d):=\left\{\gamma \in[0, \infty]^{X \times X} \mid\right.$ $\gamma \leq d\}$ is a uniform approach system on $X$.

Approach uniformities induced by a $\infty$-metric will be called metric approach uniformities.

PROPOSITION 3.2. Let $(X, \Gamma)$ be an approach uniformity. Then the following are equivalent.

(1) $(X, \Gamma)$ is a metric approach uniformity.

(2) $\sup _{\gamma \in \Gamma} \gamma \in \Gamma$

(3) $\Gamma$ is closed under the formation of (arbitrary) suprema.

We now describe metric approach uniformities in terms of uniform towers.

PROPOSITION 3.3. If $\left(\mathcal{U}_{\varepsilon}\right)_{\varepsilon}$ is the tower of a metric approach quasi-uniformity $\Gamma(d)$, then $\forall \varepsilon \in \mathbf{R}^{+}: \mathcal{U}_{\varepsilon}=\langle\{\{d<\alpha\} \mid \alpha>\varepsilon\}\rangle$.

PROOF. Immediate, since $\{d\}$ is an approach basis for $\Gamma$.

PROPOSITION 3.4. Let $(X, d)$ and $\left(Y, d^{\prime}\right)$ be $\infty$-metric spaces and let $f: X \rightarrow Y$ be a function. Then the following are equivalent. 
(1) $f:(X, d) \rightarrow\left(Y, d^{\prime}\right)$ is non-expansive

(2) $f:(X, \Gamma(d)) \rightarrow\left(Y, \Gamma\left(d^{\prime}\right)\right)$ is a uniform contraction.

This means that proposition 3.1 yields a full embedding functor pMET ${ }^{\infty} \rightarrow$ AUnif. We $^{-}$ have more.

PROPOSITION 3.5. pMET $^{\infty}$ is a full bicoreflective subcategory of AUnif.

PROOF. If $(X, \Gamma)$ is an approach uniformity, then define

$$
d_{\Gamma}:=\sup _{\gamma \in \Gamma} \gamma
$$

It is easy to verify that $d_{\Gamma}$ is a $\infty p$-metric.

Clearly id $:\left(X, \Gamma\left(d_{\Gamma}\right)\right) \rightarrow(X, \Gamma)$ is a uniform contraction, since $\forall \gamma \in \Gamma: \gamma \circ(i d \times i d) \leq \sup _{\gamma \in \Gamma} \gamma=$ $d_{\Gamma}$.

Now suppose that $f:\left(X^{\prime}, \Gamma(d)\right) \rightarrow(X, \Gamma)$ is a uniform contraction for some $\infty p$-metric $d$. Then $f:\left(X^{\prime}, \Gamma(d)\right) \rightarrow\left(X, \Gamma\left(d_{\Gamma}\right)\right)$ is a uniform contraction too, since

$$
\begin{aligned}
\xi \in \Gamma\left(d_{\Gamma}\right) & \Leftrightarrow \xi \leq \sup _{\gamma \in \Gamma} \\
& \Rightarrow \xi \circ(f \times f) \leq \sup _{\gamma \in \Gamma} \gamma \circ(f \times f) \leq d \\
& \Rightarrow \xi \circ(f \times f) \in \Gamma(d) .
\end{aligned}
$$

\subsection{UNIFORM APPROACH UNIFORMITIES}

PROPOSITION 3.6. Let $(X, \mathcal{U})$ be a uniform space. Then $\Gamma(\mathcal{U}):=\left\langle\left\{\theta_{U} \mid U \in \mathcal{U}\right\}\right\rangle$ is a uniform approach system on $X$.

PROOF. If $\mathcal{U}$ is a uniformity on $X$, then $(\mathcal{U})_{\varepsilon}$ (that is, $\mathcal{U}$ on every level), is a uniform tower. The associated uniform approach system clearly is $\Gamma(\mathcal{U})$.

Approach uniformities induced by a uniformity will be called uniform approach uniformities.

PROPOSITION 3.7. Let $(X, \Gamma)$ be an approach uniformity. Then the following are equivalent.

(1) $(X, \Gamma)$ is a uniform approach uniformity.

(2) $\Gamma=\left\langle\left\{\theta_{U} \mid U \subset X \times X\right.\right.$ s.t. $\left.\left.\theta_{U} \in \Gamma\right\}\right\rangle$

(3) $\Gamma$ has a basis consisting of functions into $\{0, \infty\}$.

Uniform approach uniformities have trivial uniform towers.

PROPOSITION 3.8. If $\left(\mathcal{U}_{\varepsilon}\right)_{\varepsilon}$ is the tower of a uniform approach uniformity $\Gamma(\mathcal{U})$, then $\forall \varepsilon \in \mathbf{R}^{+}: \mathcal{U}_{\varepsilon}=\mathcal{U}$.

PROPOSITION 3.9. Let $(X, \mathcal{U})$ and $(Y, \mathcal{V})$ be quasi-uniform spaces and let $f: X \rightarrow Y$ be a function. Then the following are equivalent.

(1) $f:(X, \mathcal{U}) \rightarrow(Y, \mathcal{V})$ is uniformly continuous

(2) $f:(X, \Gamma(\mathcal{U})) \rightarrow(Y, \Gamma(\mathcal{V}))$ is a uniform contraction. 
PROOF. We immediately see that

$$
\begin{aligned}
& f:(X, \mathcal{U}) \rightarrow(Y, \mathcal{V}) \text { is uniformly continuous } \\
\Leftrightarrow & \forall V \in \mathcal{V}, \exists U \in \mathcal{U}:(f \times f)(U) \subset V \\
\Leftrightarrow & \forall \theta_{V} \in \Gamma(\mathcal{V}), \exists \theta_{U} \in \Gamma(\mathcal{U}): \theta_{(f \times f)(U)} \geq \theta_{V} \\
\Leftrightarrow & \forall \theta_{V} \in \Gamma(\mathcal{V}), \exists \theta_{U} \in \Gamma(\mathcal{U}): \theta_{U} \geq \theta_{V} \circ(f \times f) \\
\Leftrightarrow & f:(X, \Gamma(\mathcal{U})) \rightarrow(Y, \Gamma(\mathcal{V})) \text { is a uniform contraction. }
\end{aligned}
$$

This means that proposition 3.6 yields a full embedding functor Unif $\rightarrow$ AUnif. We have more.

PROPOSITION 3.10. Unif is a bireflective and bicoreflective subcategory of AUnif.

PROOF. Let $(X, \Gamma)$ be an approach uniformity, and let $\left(\mathcal{U}_{\varepsilon}\right)_{\varepsilon}$ be its uniform tower. Then define $\mathcal{U}_{\Gamma}:=\mathcal{U}_{0}$ and $\mathcal{U}^{\Gamma}:=\bigcap_{\in \in R^{+}} \mathcal{U}_{\varepsilon}$. It is not difficult to verify that both $\mathcal{U}_{\Gamma}$ and $\mathcal{U}^{\Gamma}$ are uniformities on $X$.

The fact that id $:\left(X, \Gamma\left(\mathcal{U}_{\Gamma}\right)\right) \rightarrow(X, \Gamma)$ is a uniform contraction follows immediately from proposition 2.14. Now suppose $f:\left(X^{\prime}, \Gamma(\mathcal{U})\right) \rightarrow(X, \Gamma)$ is a uniform contraction for some uniformity $\mathcal{U}$ on $X^{\prime}$. Then $f:\left(X^{\prime}, \mathcal{U}\right) \rightarrow\left(X, \mathcal{U}_{0}\right)$ is uniformly continuous and hence $f:\left(X^{\prime}, \Gamma(\mathcal{U})\right) \rightarrow$ $\left(X, \Gamma\left(\mathcal{U}_{\mathrm{r}}\right)\right)$ is a uniform contraction.

The fact that id $:(X, \Gamma) \rightarrow\left(X, \Gamma\left(\mathcal{U}^{\Gamma}\right)\right)$ is a uniform contraction, is again a consequence of proposition 2.14. Now suppose that $f:(X, \Gamma) \rightarrow\left(X^{\prime}, \Gamma(\mathcal{U})\right)$ is a uniform contraction for some uniformity $\mathcal{U}$ on $X^{\prime}$. Then for any $\varepsilon \in \mathbf{R}^{+}$we have that $f:\left(X, \mathcal{U}_{\varepsilon}\right) \rightarrow\left(X^{\prime}, \mathcal{U}\right)$ is uniformly continuous. Hence $f:\left(X, \cap_{\varepsilon \in \mathbf{R}^{+}} \mathcal{U}_{\varepsilon}\right) \rightarrow\left(X^{\prime}, \mathcal{U}\right)$ is uniformly continuous, and thus $f:\left(X, \Gamma\left(\mathcal{U}^{\Gamma}\right)\right) \rightarrow\left(X^{\prime}, \Gamma(\mathcal{U})\right)$ is a uniform contraction.

\subsection{METRIC AND TOPOLOGICAL APPROACH SPACES}

Let $T:$ Unif $\rightarrow$ Top and $A:$ AUnif $\rightarrow$ AP be the natural forgetful functors. Then the following diagram is commutative.

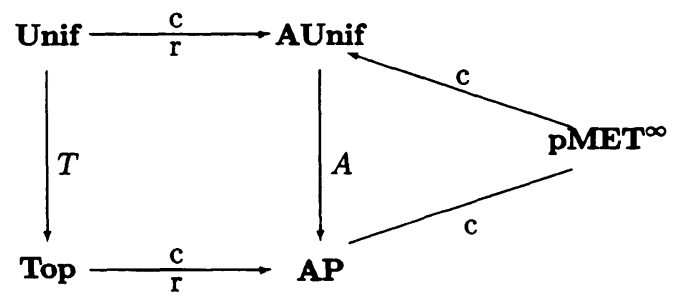

PROPOSITION 3.11. Let $(X, d)$ be a $\infty p$-metric space, then $A(\Gamma(d))=\delta_{d}$.

PROOF. Let $\delta$ denote the distance defining $A(\Gamma(d))$. Recall that $\forall x \in X, \forall A \subset X$ :

$$
\delta_{d}(x, A)=\inf _{a \in A} d(x, a) \quad \text { and } \quad \delta(x, A)=\sup _{\gamma \leq d} \inf _{a \in A} \gamma(x, a) .
$$

It is easy to see that $\delta \leq \delta_{d}$. On the other hand, we have for any $x \in X$ and $A \subset X$ that

$$
\forall a \in A, \forall \varepsilon>0, \exists \gamma \leq d: \gamma(x, a)+\varepsilon \geq d(x, a)
$$


and hence $\forall \varepsilon>0: \sup _{\gamma \leq d} \inf _{a \in A} \gamma(x, a)+\varepsilon \geq \inf _{a \in A} d(x, a)$, which, by arbitrariness of $\varepsilon$, proves the other inequality.

PROPOSITION 3.12. Let $(X, \Gamma)$ be an approach uniformity. Then the underlying topology of $\mathcal{U}^{\Gamma}$ coincides with the topological reflection of $A(\Gamma)$.

PROPOSITION 3.13. Let $(X, \Gamma)$ be an approach uniformity. Then the underlying topology of $\mathcal{U}_{\Gamma}$ coincides with the topological coreflection of $A(\Gamma)$.

\section{NATURAL EXAMPLES}

\subsection{THE FINE APPROACH UNIFORMITY}

A uniform approach space $\left(X,(\mathcal{A}(x))_{x \in X}\right)$ is (by definition) generated by a gauge of pseudometrics $\mathcal{D}$ in the sense that for all $x \in X: \mathcal{A}(x)=\langle\{d(x, \cdot) \mid d \in \mathcal{D}\}\rangle$. It is easily verified that the underlying approach space of the approach uniformity generated by $\mathcal{D}$ is again $\left(X,(\mathcal{A}(x))_{x \in X}\right)$. Therefore, every uniform approach space is compatible with some approach uniformity. There always exists a finest such structure.

We need the following preliminary result.

PROPOSITION 4.1. Let $\left(f_{\jmath}:(X, \Gamma) \rightarrow\left(X_{\jmath}, \Gamma_{\jmath}\right)\right)_{j \in J}$ be an initial AUnif-source. Then $\left(f_{j}:(X, A(\Gamma)) \rightarrow\left(X_{j}, A\left(\Gamma_{j}\right)\right)\right)_{j \in J}$ is AP-initial.

PROOF. Let $\left(\mathcal{A}_{j}(z)\right)_{z \in X}$, denote the approach systems of $A\left(\Gamma_{\jmath}\right)(j \in J)$ and let $(\mathcal{A}(x))_{x \in X}$ be the initial approach system for the given AP-source. Then $\forall z \in X_{j}$ :

$$
\mathcal{A}_{j}(z)=\left\{\gamma_{j}(z, \cdot) \mid \gamma_{j} \in \Gamma_{j}\right\}
$$

and therefore $\forall x \in X$ :

$$
\begin{aligned}
\mathcal{A}(x) & =\left\{\sup _{j \in J_{0}} \xi_{j} \circ f_{j} \mid J_{0} \subset J \text { finite, } \xi_{j} \in \mathcal{A}_{j}\left(f_{j}(x)\right)\right\} \\
& =\left\{\sup _{j \in J_{0}} \gamma_{j}\left(f_{j}(x), f_{j}(\cdot)\right) \mid J_{0} \subset J \text { finite }\right\}
\end{aligned}
$$

which clearly coincides with the approach system of $A(\Gamma)$.

DEFINITION 4.2. Let $(X, \delta)$ be a uniform approach space. Then we call $F I N E(\delta):=$ $\bigvee\{\Gamma \in|\mathbf{A U n i f}| \mid A(\Gamma)=\delta\}$ the fine approach uniformity compatible with $\delta$.

Because of the previous proposition, $A(F I N E(\delta))=\delta$.

As a matter of fact, FINE : UAP $\rightarrow$ AUnif is a functor.

PROPOSITION 4.3. Let $(X, \delta)$ and $\left(Y, \delta^{\prime}\right)$ be approach spaces and let $f: X \rightarrow Y$ be a function. If $f:(X, \delta) \rightarrow\left(Y, \delta^{\prime}\right)$ is a contraction, then $f:(X, F I N E(\delta)) \rightarrow\left(Y, F I N E\left(\delta^{\prime}\right)\right)$ is a uniform contraction.

PROOF. Let $\Gamma$ be the initial AUnif-structure for the source of all contractions $X \rightarrow$ $\left(Y, F I N E\left(\delta^{\prime}\right)\right)$. Every $\delta-\delta^{\prime}$-contraction is a $\Gamma-F I N E\left(\delta^{\prime}\right)$-uniform contraction, and thus a $A(\Gamma)$ $\delta^{\prime}$-contraction, whence $A(\Gamma)$ is finer than $\delta$.

Conversely, proposition 4.1 implies that the source of all $\delta-\delta^{\prime}$-contractions $(X, A(\Gamma)) \rightarrow\left(X, \delta^{\prime}\right)$ is initial, and therefore $A(\Gamma)$ is coarser than $\delta$. Thus $A(\Gamma)=\delta$.

$F I N E(\delta)$ is the finest structure compatible with $\delta$, thus $F I N E(\delta) \supset \Gamma$. Therefore, every $\delta-\delta^{\prime}-$ contraction is a $F I N E(\delta)-F I N E\left(\delta^{\prime}\right)$-uniform contraction. 


\subsection{SPACES OF MEASURES}

Let $X$ be a separable metrizable topological space. Let $\mathcal{M}(X)$ denote the set of all probability measures on $X$. We define for every finite set $C$ of continuous functions from $X$ into $I:=[0,1]$ (equipped with the usual topology) the map

$$
d_{C}: \mathcal{M}(X) \times \mathcal{M}(X) \rightarrow[0, \infty]:(P, Q) \mapsto \sup _{f \in C}\left|\int f d P-\int f d Q\right| .
$$

Since each $d_{C}$ is a $p$-metric, we have the following result.

PROPOSITION 4.4. $\left\{d_{C} \mid C\right.$ is a finite subset of $\left.\mathcal{C}(X, I)\right\}$ is a basis for some approach uniformity $\Gamma_{w}$ on $\mathcal{M}(X)$.

The approach uniformity $\Gamma_{w}$ in the previous proposition, shall be called the weak approach uniformity on $\mathcal{M}(X)$. The following propositions motivate this terminology.

It is a well-known result (see e.g. Billingsley [1]) that the weak topology on $\mathcal{M}(X)$ is initial for the Top-source

$$
\left(w_{f}: \mathcal{M}(X) \rightarrow[0, \infty]: P \mapsto \int f d P\right)_{f \in \mathcal{C}(X, I)}
$$

Here we have an analogous result.

PROPOSITION 4.5. The weak approach uniformity on $\mathcal{M}(X)$ is initial for the AUnifsource

$$
\left(w_{f}: \mathcal{M}(X) \rightarrow[0, \infty]: P \mapsto \int f d P\right)_{f \in \mathcal{C}(X, I)}
$$

(where $[0, \infty]$ is equipped with the usual approach uniformity).

PROOF. Let $d_{E}$ denote the euclidean metric on $[0, \infty]$. Then the initial structure of the given source is

$$
\begin{aligned}
& \left\langle\left\{\sup _{f \in C} d_{E} \circ\left(w_{f} \times w_{f}\right) \mid C \subset \mathcal{C}(X, I) \text { finite }\right\}\right\rangle \\
= & \left\langle\left\{\sup _{f \in C}\left|\int f d \cdot-\int f d \cdot\right| \mid C \subset \mathcal{C}(X, I) \text { finite }\right\}\right\rangle
\end{aligned}
$$

which is clearly the weak approach uniformity.

If $f: X \rightarrow Y$ is continuous, then $\hat{f}: \mathcal{M}(X) \rightarrow \mathcal{M}(Y)$ defined by $\hat{f}(P)(B):=P\left(f^{-1}(B)\right)$ for every Borel-set $B$ in $Y$, is continuous with respect to the weak topologies. Here we have an analogous result.

PROPOSITION 4.6. Let $X$ and $Y$ be separable metrizable topological spaces. If $f: X \rightarrow Y$ is continuous, then $\hat{f}:\left(\mathcal{M}(X), \Gamma_{w}^{X}\right) \rightarrow\left(\mathcal{M}(Y), \Gamma_{w}^{Y}\right)$ is a uniform contraction.

PROOF. For every $C \subset \mathcal{C}(Y, I)$ finite, we have that $d_{C} \circ(\hat{f} \times \hat{f}) \in \Gamma_{w}^{X}$ since $\forall P, Q \in \mathcal{M}(X)$ :

$$
\begin{aligned}
d_{C}(\hat{f}(P), \hat{f}(Q)) & =\sup _{g \in C}\left|\int g d \hat{f}(P)-\int g d \hat{f}(Q)\right| \\
& =\sup _{g \in C}\left|\int g \circ f d P-\int g \circ f d Q\right| .
\end{aligned}
$$

In Lowen [9] the set $\mathcal{M}(X)$ is equipped with the so-called weak approach distance.

PROPOSITION 4.7. The underlying distance of the weak approach uniformity is the weak distance. 


\subsection{HYPERSPACES}

Let $(X, d)$ be a metric space. The collection of all non-empty closed subsets of $X$ is denoted by $C L(X)$. For every finite subset $F \subset X$ we define

$$
d_{F}: C L(X) \times C L(X) \rightarrow[0, \infty]:(A, B) \mapsto \sup _{x \in F}|d(x, A)-d(x, B)|
$$

Since all $d_{F}$ are $p$-metrics, we have the following result.

PROPOSITION 4.8. The collection $\left\{d_{F} \mid F \in 2^{(X)}\right\}$ is a basis for some appraach uniformity on $C L(X)$.

The approach uniformity in the previous proposition shall be referred to as the Wijsman approach uniformity on $C L(X)$. The following propositions motivate this terminology and enhance the canonicity of the example.

It is known (see e.g. Beer [3]) that the Wijsman topology on $C L(X)$ is initial for the Topsource

$$
(d(x, \cdot): C L(X) \rightarrow[0, \infty]: A \mapsto d(x, A))_{x \in X} .
$$

In AUnif we have an analogous result.

PROPOSITION 4.9. The Wijsman approach uniformity is initial for the source

$$
(d(x, \cdot): C L(X) \rightarrow[0, \infty]: A \mapsto d(x, A))_{x \in X}
$$

(where $[0, \infty]$ is equipped with the usual approach uniformity).

PROOF. Let $d_{E}$ denote the euclidean metric on $[0, \infty]$. The initial structure for the given source has a basis consisting of functions

$$
\sup _{x \in F} d_{E} \circ(d(x, \cdot) \times d(x, \cdot))=\sup _{x \in F}|d(x, \cdot)-d(x, \cdot)|
$$

where $F \in 2^{(X)}$, which clearly determine the Wijsman approach uniformity.

The Wijsman topology on $C L(X)$ is also initial for the singleton source

$$
C L(X) \rightarrow[0, \infty]^{X}: A \mapsto d(\cdot, A)
$$

where $[0, \infty]^{X}$ is equipped with the product topology (see Beer [3]).

In AUnif too, we have the following.

PROPOSITION 4.10. The Wijsman approach uniformity on $C L(X)$ is initial for the singleton source

$$
\phi: C L(X) \rightarrow[0, \infty]^{X}: A \mapsto d(\cdot, A)
$$

where $[0, \infty]^{X}$ is equipped with the product approach uniformity.

PROOF. Let $d_{E}$ denote the euclidean metric on $[0, \infty]$. The approach uniformity on $[0, \infty]^{X}=\prod_{x \in X}[0, \infty]$ has a basis consisting of functions of the form

$$
\sup _{x \in F} d_{E} \circ\left(\pi_{x} \times \pi_{x}\right)
$$

where $F \in 2^{(X)}$. The initial structure of the given source is therefore determined by functions of the form

$$
\sup _{x \in F} d_{E} \circ\left(\pi_{x} \times \pi_{x}\right) \circ(\phi \times \phi)
$$


We finish the proof by noticing that $\forall A, B \in C L(X)$ :

$$
\begin{aligned}
& \sup _{x \in F} d_{E} \circ\left(\pi_{x} \times \pi_{x}\right) \circ(\phi \times \phi)(A, B) \\
= & \sup _{x \in F}|d(x, A)-d(x, B)| .
\end{aligned}
$$

The following result also illustrates that the definition for the Wijsman approach uniformity is natural.

PROPOSITION 4.11. The $\infty$-metric coreflection of the Wijsman approach uniformity on $C L(X)$ is the Hausdorff-metric $h_{d}$.

PROOF. We have $\forall A, B \in C L(X)$ :

$$
\sup _{F \in 2^{(X)}} d_{F}(A, B)=\sup _{x \in X} d_{\{x\}}(A, B)=h_{d}(A, B) .
$$

On the analogy of the definitions in the topological and the uniform case, we define the following notion of admissibility.

DEFINITION 4.12. Let $(X, d)$ be a metric space. An appoach uniformity $\Gamma$ on $C L(X)$ is called admissible iff $\psi:(X, \Gamma(d)) \rightarrow(C L(X), \Gamma): x \mapsto\{x\}$ is a well-defined embedding.

While the Wijsman topology and the Wijsman distance are always admissible, this is not the case for the Wijsman uniformity and for the Wijsman approach uniformity. But we do have the following partial result.

PROPOSITION 4.13. If $(X, d)$ is totally bounded, then the Wijsman approach uniformity is admissible.

PROOF. Since the underlying topology of $(X, d)$ is Hausdorff, $\psi$ (as in definition 4.12) is well-defined.

Clearly

$$
\begin{aligned}
d_{F}(\{x\},\{y\}) & =\sup _{z \in F}|d(x, z)-d(y, z)| \\
& \leq \sup _{z \in F} d(x, y) \\
& =d(x, y)
\end{aligned}
$$

Conversely, fix $\varepsilon>0$ and let $F \in 2^{(X)}$ be such that $\bigcup_{z \in F} B_{d}(z, \varepsilon / 2)=X$. We shall show that

$$
\forall x, y \in X: d(x, y) \leq d_{F}(\{x\},\{y\})+\varepsilon .
$$

Let $x, y \in X$, and choose $z \in F$ such that $d(y, z) \leq \varepsilon / 2$. If $d(x, y) \leq \varepsilon$, then there is nothing to prove. If $d(x, y)>\varepsilon / 2$, then

$$
\begin{aligned}
d(x, y) & \leq d(x, z)+d(y, z) \\
& \leq d(x, z)-d(y, z)+\varepsilon \\
& \leq \sup _{z \in F}|d(x, z)-d(y, z)|+\varepsilon .
\end{aligned}
$$

The following example shows that the total boundedness is a necessary condition.

EXAMPLE 4.14. Let $d$ be the usual metric on $\mathbf{R}^{2}$ and let $\Gamma_{W}$ denote the Wijsman approach uniformity on $C L\left(\mathbf{R}^{2}\right)$. Then $\psi:\left(\mathbf{R}^{2}, \Gamma(d)\right) \rightarrow\left(C L\left(\mathbf{R}^{2}\right), \Gamma_{W}\right): x \mapsto\{x\}$ is not an embedding. 


\subsection{FUNCTION SPACES}

Let $X$ be a set. Let $\mathcal{X}$ be a collection of subsets of $X$ such that $\mathcal{X}$ covers $X$ and such that $\mathcal{X}$ is closed under the formation of finite unions. Let $(Y, d)$ be a metric space. For any $A \in \mathcal{X}$, we define

$$
d_{A}: Y^{X} \times Y^{X} \rightarrow[0, \infty]:(f, g) \mapsto \sup _{x \in A} d(f(x), g(x)) .
$$

Since each $d_{A}$ is a $p$-metric we have the following result.

PROPOSITION 4.15. $\Gamma_{\mathcal{X}}:=\left\langle\left\{d_{A} \mid A \in \mathcal{X}\right\}\right\rangle$ is an approach uniformity on $Y^{X}$.

Let us start with an example.

PROPOSITION 4.16. If $\mathcal{X}=2^{(X)}$, then $\left(Y^{X}, \Gamma_{\mathcal{X}}\right)$ coincides with the AUnif-product $\left(Y^{X}, \Pi_{x \in X} \Gamma(d)\right)$.

PROOF. The uniform approach system of $\prod_{x \in X} \Gamma(d)$ is given by

$$
\left\langle\left\{\sup _{x \in A} d \circ\left(\pi_{x} \times \pi_{x}\right) \mid A \in 2^{(X)}\right\}\right\rangle
$$

which clearly coincides with $\Gamma_{\mathcal{X}}$.

The uniform and the metric coreflection of $\Gamma_{\mathcal{X}}$ are well-known.

PROPOSITION 4.17. The uniform coreflection of $\Gamma_{\mathcal{X}}$ is the uniformity of $\mathcal{X}$-convergence.

PROOF. This is clear, since the uniformity of $\mathcal{X}$-convergence has a basis consisting of entourages of the form

$$
\left\langle\left\{\left\{(f, g) \in Y^{X} \times Y^{X} \mid \sup _{x \in A} d(f(x), g(x))<\varepsilon\right\} \mid A \in \mathcal{X}, \varepsilon>0\right\}\right\rangle .
$$

PROPOSITION 4.18. The $\infty p$-metric coreflection of $\Gamma_{\mathcal{X}}$ is the uniform metric.

PROOF. If $f, g \in Y^{X}$, then

$$
\begin{aligned}
d\left(\Gamma_{\mathcal{X}}\right)(f, g) & =\sup _{\boldsymbol{A} \in \mathcal{X}} \sup _{x \in A} d(f(x), g(x)) \\
& =\sup _{\boldsymbol{x} \in \boldsymbol{X}} d(f(x), g(x)) .
\end{aligned}
$$

Let $k_{y}$ denote the map that always takes the value $y$.

PROPOSITION 4.19. The map $(Y, \Gamma(d)) \rightarrow\left(Y^{X}, \Gamma_{\mathcal{X}}\right): y \mapsto k_{y}$ is an embedding.

PROOF. For arbitrary $A \in \mathcal{X}$ we have

$$
\begin{aligned}
d_{A}\left(k_{x}, k_{y}\right) & =\sup _{z \in A} d\left(k_{x}(z), k_{y}(z)\right) \\
& =d(x, y) .
\end{aligned}
$$

\subsection{IMPLEMENTATION OF $p q$-METRICS}

qMET is considered to be a suitable category for domain theory. A well-known example is the quasi-metric

$$
d: X \times X \rightarrow \mathbf{R}^{+}: \begin{cases}(x, y) \mapsto 0 & \text { if } x \sqsubseteq y \\ (x, y) \mapsto 2^{-k} & \text { if } x \nsubseteq y \text { and } k=\min \left\{n \mid x_{n} \neq y_{n}\right\}\end{cases}
$$

on $X=\{0,1\}^{*}$, the set of all infinite strings of zero's and one's. Recall that $x \sqsubseteq y$ means " $x$ is a prefix of $y$ ". 
If one wants to implement $d$, one will not implement the function $d$ itself, since its domain consists of infinite (!) strings. One would rather implement a series of test functions $\left(f_{n}\right)_{n}$ on $X \times X$, that check whether $d(x, y) \leq 2^{-n}$ or not, say

$$
f_{n}: X \times X \rightarrow\{0, \infty\}:\left\{\begin{array}{lll}
(x, y) \mapsto 0 & \text { if } d(x, y) \leq 2^{-n} \\
(x, y) \mapsto \infty & \text { otherwise. }
\end{array}\right.
$$

PROPOSITION 4.20. $\left\{f_{n} \mid n \in \mathbf{N}_{0}\right\}$ is a basis for an approach quasi-uniformity $\Gamma$ on $X$.

PROOF. This follows from the observations that $\forall n, m \in \mathbf{N}_{0}: f_{n} \vee f_{m}=f_{n \vee m}$ and $\forall n \in \mathbf{N}_{0}: \forall x, y, z \in X: f_{n}(x, z) \leq f_{n+1}(x, y)+f_{n+1}(y, z)$.

The quasi-uniform coreflection of $\Gamma$ is the quasi-uniformity $\mathcal{U}_{d}$, which was studied by e.g. Smyth (Smyth [10]) and Sünderhauf (Sünderhauf [11]).

The $p q \infty$-metric coreflection $d_{\Gamma}$ expresses the prefix-order, in the sense that $d_{\Gamma}(x, y)=0 \Leftrightarrow$ $x \sqsubseteq y$, since

$$
\sup _{n} f_{n}(x, y)=0 \Leftrightarrow \forall n: d(x, y)<2^{-n} \Leftrightarrow d(x, y)=0
$$

\section{ACKNOWLEDGMENT.}

The second author is Aspirant of the Foundation for Scientific Research Flanders.

\section{REFERENCES}

[1] BILLINGSLEY, P., Convergence of probability measures, John Wiley \& Sons, 1968

[2] BAUER, H., Probability theory and elements of measure theory, Holt, Rinehart \& Winston inc., 1972

[3] BEER, G. and LUCHETTI, R., Weak topologies for the closed subsets of a metrizable space, Trans. Amer. Math. Soc. 335, 1993, 805-822

[4] ČECH, E., Topological spaces, John Wiley \& Sons, 1966

[5] ISBELL, J.R., Uniform spaces, vol. 12 of Math. Surveys, Amer. Math. Soc., 1964

[6] FLETCHER, P. and LINDGREN, W.F., Quasi-uniform spaces, Lecture Notes in Pure and Applied Mathematics 77, Dekker, 1982

[7] KUNZI, H.P.A., Functorial admissible quasi-uniformities on topological spaces, Top. Appl. 43, 1992, 27-36

[8] LOWEN, R., Approach spaces: a common supercategory of TOP and MET, Math. Nachrichten 141 (1989), 183-226

[9] LOWEN, R., Approach spaces, Oxford Mathematical Monographs, Oxford University Press, 1996

[10] SMYTH, M.B., Quasi-uniformities: reconciling domains with metric spaces. In: Third Workshop on Mathematical Foundations of Programming Language Semantics, Lecture Notes in Computer Science, vol. 298, Springer Verlag, 1987

[11] SUNDERHAUF, PH., A faithful computational model of the real numbers, preprint, 1994 


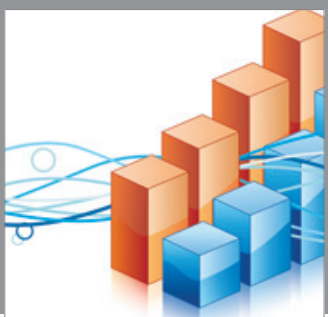

Advances in

Operations Research

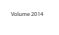

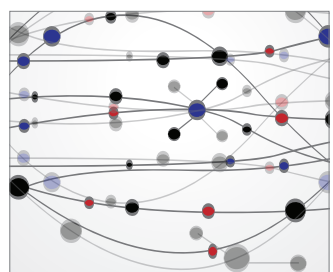

\section{The Scientific} World Journal
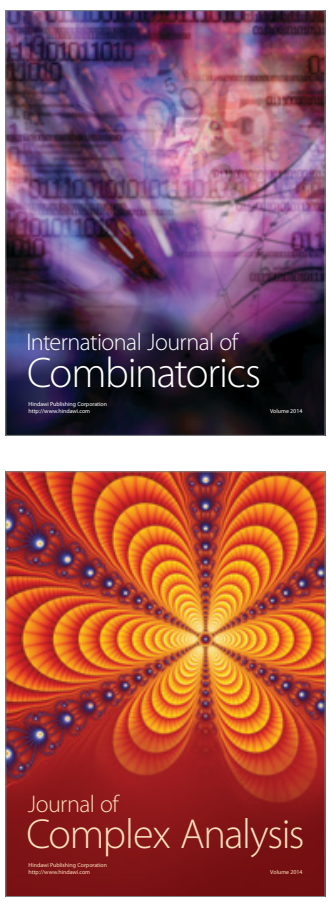

International Journal of

Mathematics and

Mathematical

Sciences
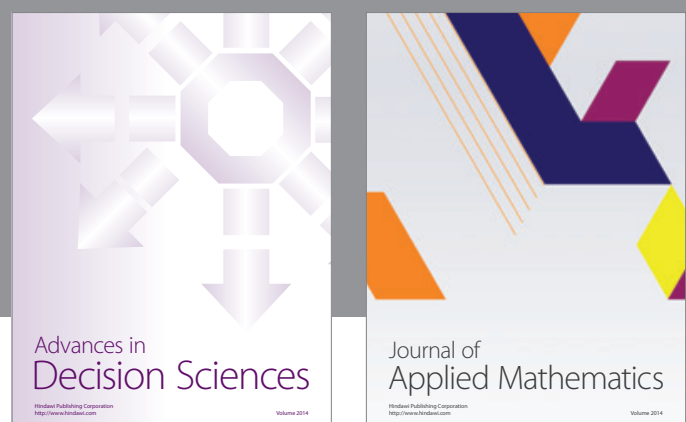

Journal of

Applied Mathematics
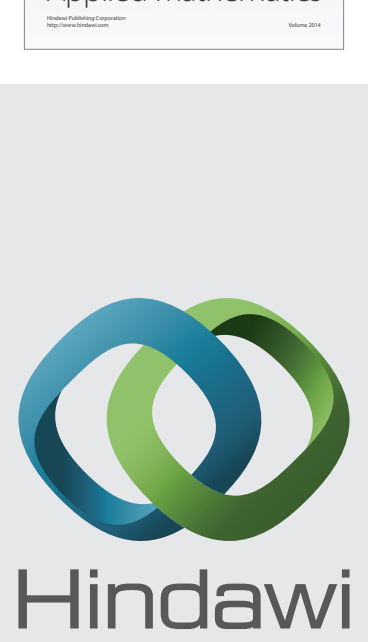

Submit your manuscripts at http://www.hindawi.com
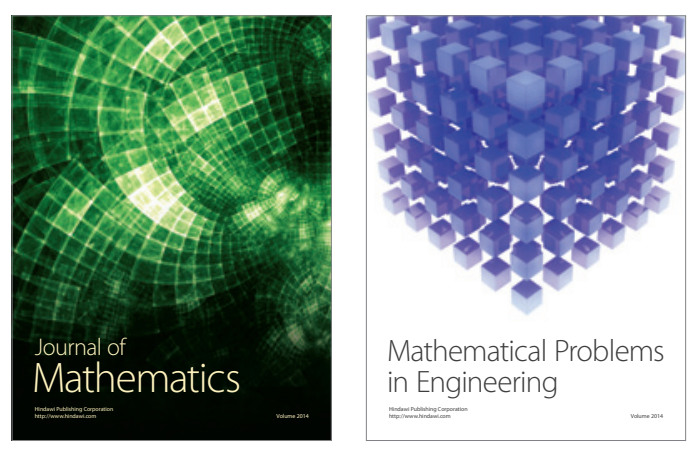

Mathematical Problems in Engineering
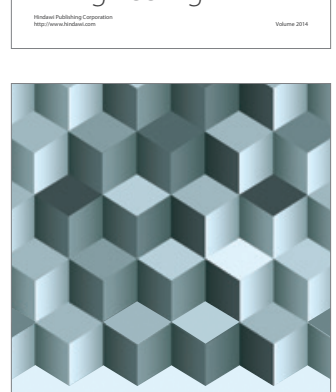

Journal of

Function Spaces
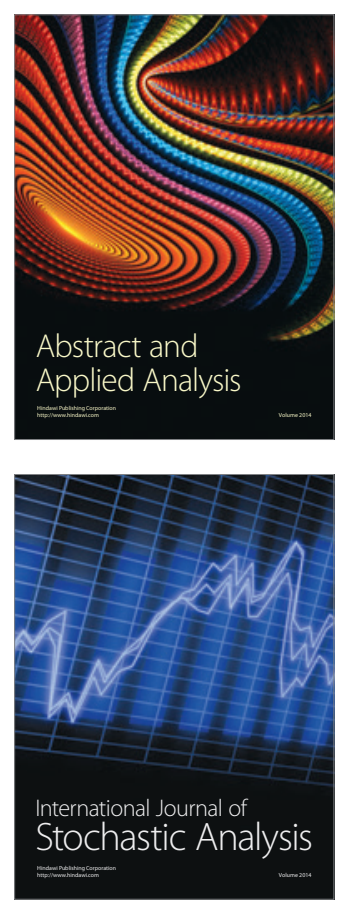

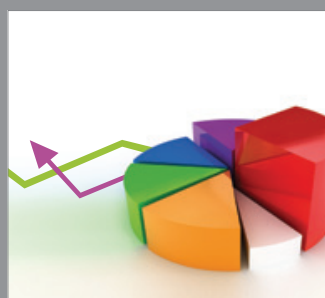

ournal of

Probability and Statistics

Promensencen
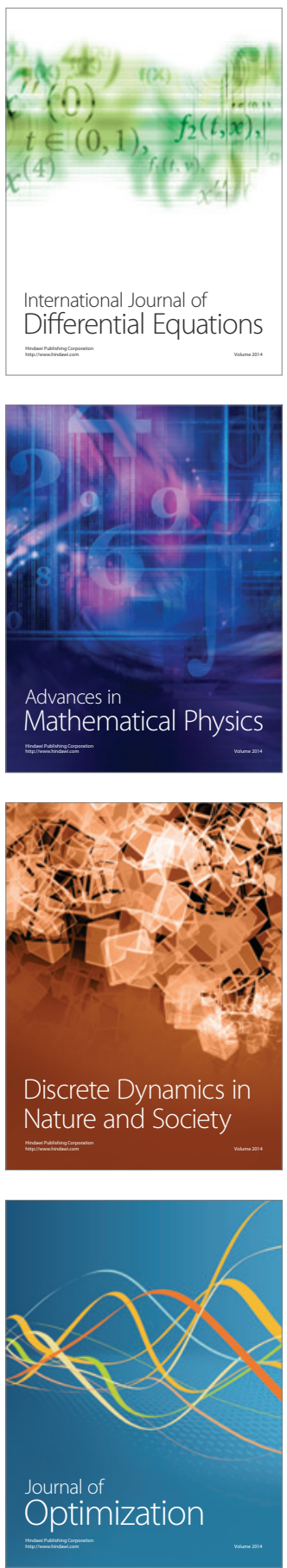